\title{
Real-world hematological adverse events in Chinese patients with advanced ovarian cancer treated with an individualized starting dose of niraparib
}

\author{
Junjian Wang^, Jianqing Zhu^ \\ Department of Gynecologic Oncology, Cancer Hospital of the University of Chinese Academy of Sciences (Zhejiang Cancer Hospital), Hangzhou, \\ China \\ Contributions: (I) Conception and design: J Wang; (II) Administrative support: J Zhu; (III) Provision of study materials or patients: J Wang; (IV) \\ Collection and assembly of data: J Wang; (V) Data analysis and interpretation: J Wang; (VI) Manuscript writing: Both authors; (VII) Final approval \\ of manuscript: Both authors. \\ Correspondence to: Prof. Jianqing Zhu. Department of Gynecologic Oncology, Cancer Hospital of the University of Chinese Academy of Sciences \\ (Zhejiang Cancer Hospital), No. 1, Banshan East Road, Hangzhou 310022, China. Email: zjq-hz@126.com.
}

Background: This work set out to examine the hematological adverse events (AEs) of an individualized starting dose (ISD) of niraparib in Chinese patients with ovarian cancer (OC).

Methods: The medical records of 43 patients with OC who were treated with an ISD of niraparib at the Cancer Hospital of The University of Chinese Academy of Sciences between February 2019 and January 2020 were retrospectively reviewed. Treatment-emergent hematological AEs were analyzed.

Results: Of the 43 patients with OC, 28 (65.1\%) had hematological AEs of $\geq$ grade 1 , including thrombocytopenia (39.5\%), leukopenia (37.2\%), and anemia (34.9\%). Ten $(23.3 \%)$ patients developed grade 3/4 hematological AEs, including thrombocytopenia (11.6\%), leukopenia (9.3\%), and anemia (7.0\%). Among the individuals who developed AEs during treatment, 9 (32.1\%) patients had their treatment interrupted, with treatment being restarted in 8 (28.6\%) cases, and 4 (14.3\%) patients had the drug dose decreased. No deaths were reported. The median times to the occurrence of any-grade leukopenia, anemia, and thrombocytopenia were 30 (range, 7 to 162), 34 (range, 7 to 108), and 20 (range, 13 to 180) days, respectively. Most AEs occurred within the first 3 months of treatment (93.8\% leukopenia, 80.0\% anemia, and $76.5 \%$ thrombocytopenia). Treatments for AEs included supplementation of recombinant human granulocyte colony-stimulating factor $(\mathrm{n}=5,17.9 \%)$, erythrocytes $(\mathrm{n}=2,7.1 \%)$, and recombinant human thrombopoietin ( $\mathrm{n}=5,17.9 \%)$.

Conclusions: The incidence of adverse hematological reactions to an ISD of niraparib in Chinese patients with advanced OC is relatively lower in the real world than in the phase III clinical trials PRIMA (also an ISD) and NOVA. These hematological AEs can be managed through dose adjustment and symptomatic therapy.

Keywords: Ovarian cancer (OC); platinum-based chemotherapy; niraparib; real-world study; adverse events (AEs)

Submitted Apr 08, 2021. Accepted for publication May 17, 2021.

doi: 10.21037/atm-21-2252

View this article at: http://dx.doi.org/10.21037/atm-21-2252

^ ORCID: Junjian Wang, 0000-0002-3023-3329; Jianqing Zhu, 0000-0001-5736-7397. 


\section{Introduction}

Ovarian cancer (OC) affects more women than any other malignancy of the female reproductive tract. Claiming the lives of approximately 22,500 Chinese women each year, the disease poses a severe threat to public health (1). At diagnosis, about $70 \%$ of cases already show advanced disease $(2,3)$. The preferred treatment for OC is surgery combined with platinum-based chemotherapy $(2,3)$. Although clinical remission can be achieved after the initial treatment, most OC cases relapse within 3 years, and 5 -year survival is only about $40-50 \%(4,5)$. The focus of clinical management of OC is to prolong the efficacy of platinum-containing chemotherapy and delay recurrence $(2,3)$.

Niraparib represents a known poly-ADP-ribose polymerase (PARP 1/2) suppressor. PARP inhibitors can block the repair of DNA single-strand breaks by PARP. Loss of homologous recombination repair function caused by mutations in the breast cancer susceptibility genes BRCA1 and BRCA2 combined with PARP inhibition exerts a synergistic synthetic lethal effect that results in tumor cell death $(6,7)$. At the same time, PARP inhibitors suppress the reversible dissociation of the PARP protein from DNA, which leads to long-term DNA-PARP complex stability and inhibits the subsequent DNA repair process, causing tumor cell death (6-8). Based on the NOVA study (9), niraparib was approved by the United States Food and Drug Administration (FDA) in 2017 as maintenance therapy for platinum-sensitive recurrent epithelial OC (EOC), fallopian tube cancer (FTC), and primary peritoneal cancer (PPC) in adult patients with a complete response (CR) or partial response (PR) to platinum-containing chemotherapeutic regimens (2). In 2020, niraparib was approved by the National Medical Products Administration (NMPA) for first-line maintenance therapy or maintenance therapy after recurrence in adult patients with advanced EOC, FTC, or PPC with CR or PR to platinum-containing chemotherapeutic regimens, irrespective of BRCA mutation status.

The ENGOT-OV16/NOVA (9), PRIMA (10), and NORA (11) trials showed that niraparib can significantly improve progression-free survival (PFS) in patients with OC in comparison with placebo. In the above trials, the commonest grade 3/4 adverse events (AEs) after niraparib treatment included thrombocytopenia (31.0-33.8\%), anemia (25.3-28.7\%), and neutropenia (12.8-19.6\%) $(9,10)$. In the ENGOT-OV16/NOVA trial (9), nausea, thrombocytopenia, and fatigue occurred in $73.6 \%, 61.3 \%$, and $59.4 \%$ of cases, respectively. However, a real-world study (12) showed that the 3 most common all-grade AEs in the first 3 months post-niraparib initiation were fatigue (24\%; grade $3 / 4$, $3 \%$ ), nausea (16\%; grade $3 / 4,2 \%)$, and thrombocytopenia (14\%; grade $3 / 4,3 \%$ ). Hence, important discrepancies can be seen in safety data between clinical trials and real-world studies, and real-world safety data in Chinese populations are lacking. Real-world studies are more likely to represent the actual clinical reality than clinical trials, for which many patients are not eligible $(13,14)$, and both study types are considered to be complementary.

Therefore, this real-world study aimed to explore hematological AEs and management strategies in Chinese patients with OC treated with an individualized starting dose (ISD) of niraparib. We present the following article in accordance with the STROBE reporting checklist (available at http://dx.doi.org/10.21037/atm-21-2252).

\section{Methods}

\section{Patients}

This real-world study retrospectively analyzed the data of patients with OC who received an ISD of niraparib between February 2019 and January 2020 at the Cancer Hospital of The Chinese Academy of Sciences. All procedures performed in this study involving human participants were in accordance with the Declaration of Helsinki (as revised in 2013). The study was approved by institutional ethics board of Cancer Hospital of the University of Chinese Academy of Sciences (Zhejiang Cancer Hospital) (No. IRB-2021-71), and the requirement for informed consent was waived.

The inclusion criteria for patients were: (I) age $>18$ years; (II) a diagnosis of EOC, ETC, or PPC; (III) CR or PR to a platinum-containing chemotherapeutic regimen; and (IV) niraparib used as maintenance or later-line treatment. The exclusion criterion was incomplete data.

\section{Data collection and definitions}

Baseline data obtained from patient clinical records included age, weight, tumor classification, International Federation of Gynecology and Obstetrics (FIGO) stage, Eastern Cooperative Oncology Group (ECOG) performance status, BRCA mutation status, type of surgery, response to previous platinum-based chemotherapy, type of niraparib therapy, cancer antigen 125 (CA-125) levels, AEs, and time to $\mathrm{AE}$ occurrence. Myelosuppression-related AEs included 
Table 1 Baseline characteristics of patients before niraparib initiation at $200 \mathrm{mg} /$ day

\begin{tabular}{|c|c|}
\hline Characteristics & Total $(n=43)$ \\
\hline Age, years, median [IQR] & $57[52-64]$ \\
\hline Weight, $\mathrm{kg}$, mean \pm standard deviation & $50.9 \pm 8.4$ \\
\hline Weight, kg, median [range] & $50.5[33-75]$ \\
\hline \multicolumn{2}{|l|}{ Type of cancer, n (\%) } \\
\hline EOC & $40(93.0)$ \\
\hline FTC & $1(2.3)$ \\
\hline PPC & $2(4.7)$ \\
\hline \multicolumn{2}{|l|}{ FIGO, n (\%) } \\
\hline I & $1(2.4)$ \\
\hline II & $3(7.1)$ \\
\hline III & $22(52.4)$ \\
\hline IV & $16(38.1)$ \\
\hline \multicolumn{2}{|l|}{ ECOG performance status, $n(\%)$} \\
\hline 0 & $36(83.7)$ \\
\hline 1 & $6(14.0)$ \\
\hline 2 & $1(2.3)$ \\
\hline \multicolumn{2}{|l|}{$B R C A$ mutation status, $\mathrm{n}(\%)$} \\
\hline Wild type & $27(62.8)$ \\
\hline Mutant & $14(32.6)$ \\
\hline Unknown & $2(4.6)$ \\
\hline
\end{tabular}

Response to most recent platinum-containing chemotherapy, $\mathrm{n}(\%)$

CR

9 (20.9)

PR

$19(44.2)$

SD

$15(34.9)$

Type of previous surgery, $\mathrm{n}(\%)$

Primary debulking surgery

$30(69.8)$

NACT and interval debulking surgery

$12(27.9)$

Unknown

$1(2.3)$

Niraparib therapy, $\mathrm{n}(\%)$

First-line maintenance therapy

Later-line therapy

Maintenance therapy for platinum-sensitive recurrent patients

$4(9.3)$

$\mathrm{CA}-125, \mathrm{U} / \mathrm{mL}$

$23.3(8.4,129.2)$

IQR, interquartile range; EOC, epithelial ovarian cancer; FTC, fallopian tube cancer; PPC, primary peritoneal cancer; FIGO, International Federation of Gynecology and Obstetrics; ECOG, Eastern Cooperative Oncology Group; CR, complete response; $\mathrm{PR}$, partial response; SD, stable disease; NACT, neoadjuvant chemotherapy; CA-125, cancer antigen 125. any $\mathrm{AE}$, leukopenia, anemia, and thrombocytopenia. All myelosuppression-related AEs were categorized according to Common Terminology Criteria for Adverse Events (CTCAE) 4.0.3.

\section{Statistical analysis}

Statistical analyses were carried out with SPSS 22.0 (SPSS, USA). Continuous data conforming to a normal distribution, as determined by the Kolmogorov-Smirnov test, were expressed as mean \pm standard deviation, and those with skewed distribution as median [range or interquartile range (IQR)]. Categorical variables were expressed as $\mathrm{n}(\%)$.

\section{Results}

\section{Clinicodemographic features}

In total, 43 patients who initiated niraparib at $200 \mathrm{mg} / \mathrm{day}$ were enrolled in this study. The median follow-up time was 2 (range, $0.2-11.8$ ) months. The cohort had a median age of 57 (IQR, 52-64) years and an average weight of $50.9 \pm 8.4$ (range, 33-75) kg. The primary tumor type was EOC, which accounted for 40 (93\%) cases. Among them, 22 (52.4\%) and 16 (38.1\%) cases were FIGO stages III and IV, respectively. BRCA wild type and mutants were detected in $27(62.8 \%)$ and 14 (32.6\%) patients, respectively. Primary debulking surgery was the most common type of surgery $(\mathrm{n}=30 ; 69.8 \%)$, followed by neoadjuvant chemotherapy (NACT) combined with interval debulking surgery (Table 1).

\section{Niraparib treatment}

At niraparib initiation, a higher proportion of cases had achieved PR ( $\mathrm{n}=19 ; 44.2 \%)$ than stable disease (SD) $(\mathrm{n}=15$; $34.9 \%)$ or CR ( $\mathrm{n}=9 ; 20.9 \%)$ following platinum-containing chemotherapeutic regimens. Among the entire cohort, $25(58.1 \%), 14(32.6 \%)$, and $4(9.3 \%)$ patients received niraparib as maintenance therapy in the first-line setting, as later-line treatment, and as maintenance therapy for platinum-sensitive recurrent disease, respectively (Table 1).

\section{AEs}

A total of $28(65.1 \%)$ cases experienced $\geq$ grade 1 hematological AEs, including thrombocytopenia (39.5\%), leukopenia (37.2\%), and anemia (34.9\%). Ten (23.3\%) cases developed hematological AEs of grade 3/4, including 


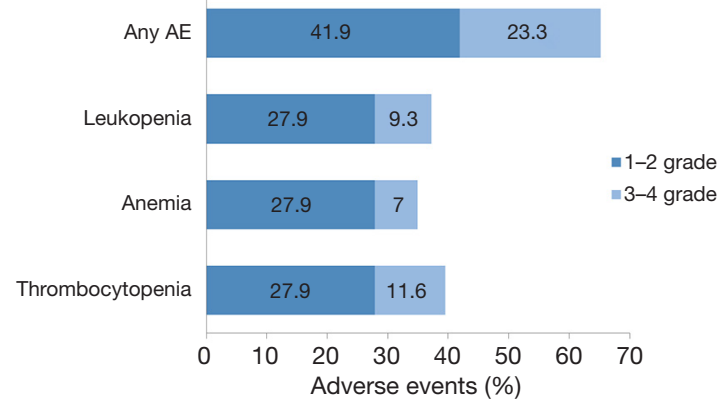

Figure 1 Myelosuppression AEs. AEs, adverse events.

Table 2 Overall summary of any-grade AEs in the study population

\begin{tabular}{lc}
\hline AEs & Total $(n=43)$ \\
\hline Any related AEs, $n(\%)$ & $28(65.1)$ \\
Any related CTCAE grade $\geq 3$ AE, $n(\%)$ & $10(23.3)$ \\
Any AE leading to dose reduction & $4 / 28(14.3)^{\star}$ \\
Any AE resulting in treatment interruption & $9 / 28(32.1)^{\star}$ \\
Any AE resulting in treatment discontinuation & $1(2.3)$ \\
Any AE resulting in death & 0
\end{tabular}

*, percentage of the 28 cases with AEs. AE, adverse event; CTCAE, Common Terminology Criteria for Adverse Events.

thrombocytopenia (11.6\%), leukopenia (9.3\%), and anemia (7.0\%) (Figure 1). Among the 28 patients who developed AEs during the treatment period, 9 patients had their treatment interrupted $(32.1 \%)$, with $8(3.6 \%)$ cases had restarted niraparib therapy after treatment for the AEs, and $4(14.3 \%)$ patients had their drug dose reduced (Table 2).

\section{Timing of AE occurrence}

The median times to the occurrence of any-grade leukopenia, anemia, and thrombocytopenia were 30 (range, 7 to 162), 34 (range, 7 to 108), and 20 (range, 13 to 180 ) days, respectively (Figure 2). Most AEs occurred within the first 3 months of treatment (93.8\% leukopenia, 80.0\% anemia, and $76.5 \%$ thrombocytopenia) (Table 3).

\section{AE management}

Treatments for AEs included supplementation of recombinant human granulocyte colony-stimulating factor $(n=5,17.9 \%)$, erythrocytes $(n=2,7.1 \%)$, and recombinant human thrombopoietin $(\mathrm{n}=5,17.9 \%)$.

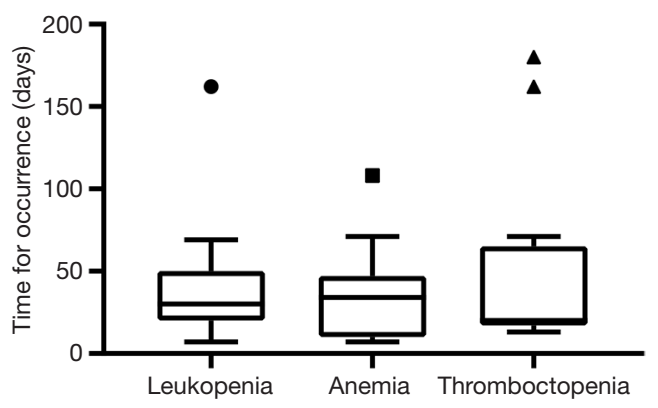

Figure 2 Time to occurrence of any-grade myelosuppression AEs. AEs, adverse events.

Table 3 Timing to the occurrence of myelosuppression AEs

\begin{tabular}{lcc}
\hline \multirow{2}{*}{ AEs } & \multicolumn{2}{c}{ Grade $\geq 1$ AE, $n(\%)$} \\
\cline { 2 - 3 } & $0-3$ months & 3-6 months \\
\hline Leukopenia $(n=16)$ & $15(93.8)$ & $1(6.3)$ \\
Anemia $(n=15)$ & $12(80.0)$ & $3(20.0)$ \\
Thrombocytopenia $(n=17)$ & $13(76.5)$ & $4(23.5)$ \\
\hline
\end{tabular}

$\mathrm{AE}$, adverse event.

\section{Discussion}

OC is the leading cause of death among all gynecological cancers in developed countries, with most patients presenting with FIGO stage III and IV (3). Treatment strategy of OC has dramatically changed over the last decade both in the new diagnosed and recurrent OC patients. PARP inhibit would target tumors with defects in the HR pathway due to $B R C A 1$ or $B R C A 2$ mutations but have little toxicity on normal cells with functional HR, which could lead to more favorable outcomes in OC (6). Maintenance treatment is currently able to delay disease progression with manageable toxicity. $B R C A 1 / 2$ mutations and homologous recombination deficiency (HRD) are the main predictive biomarkers for the majority of PARP inhibitors. PARP inhibitors have transformed OC from a "killer" to a chronic disease.

Data available from Western populations suggest discrepancies in hematological AEs between randomized controlled trials and real-world studies $(9,10,12,15)$. Therefore, this study aimed to examine hematological AEs and management strategies in Chinese patients with OC who received an ISD of niraparib. The results strongly suggested that most hematological AEs in patients with 
advanced OC could be managed by dose modification and/ or symptomatic treatment, with only $2.3 \%$ of patients discontinuing the treatment. Thus, niraparib administration with an ISD is a tolerable option for advanced OC.

The ENGOT-OV16/NOVA trial (9) showed that niraparib could significantly improve PFS in patients with germinal $B R C A$-mutated OC [21.0 vs. 5.5 months, hazard ratio $(\mathrm{HR})=0.27 ; 95 \%$ confidence interval $(\mathrm{CI}): 0.17$ to 0.41 ; $\mathrm{P}<0.001$, patients without germinal $B R C A$-mutated OC and with HRD (12.9 vs. 3.8 months; $\mathrm{HR}=0.38$; 95\% CI: 0.24 to $0.59 ; \mathrm{P}<0.001$ ), and those without a germline $B R C A$ mutation (9.3 vs. 3.9 months; HR $=0.45$; $95 \%$ CI: 0.34 to $0.61 ; \mathrm{P}<0.001)$ compared with placebo-treated cases. In the PRIMA trial (10), patients with HRD OC had an improved median PFS duration (21.9 vs. 10.4 months, HR $=0.43 ; 95 \%$ CI: 0.31 to $0.59 ; \mathrm{P}<0.001)$. The randomized, double-blind, controlled, phase 3 trial (NORA, niraparib for maintenance treatment) involved Chinese individuals with platinumsensitive recurrent $\mathrm{OC}$ with germline $B R C A$ mutation or high-grade serious histologic features who attained a CR or PR with platinum-containing chemotherapy (11). In the NORA trial, niraparib reduced the odds of tumor progression in the general patient population, with an improved median PFS (HR $=0.32$; $95 \%$ CI: 0.23 to 0.45 , $\mathrm{P}<0.0001)$. These trials showed a survival benefit of niraparib for patients with OC; but, in the clinical setting, safety must also be considered.

A recent retrospective assessment of data from the ENGOT-OV16/NOVA study (15) showed that individuals with a body weight $<77 \mathrm{~kg}$ or platelets $<150,000 / \mu \mathrm{L}$ at baseline might benefit from an ISD of $200 \mathrm{mg} /$ day. An ISD should be used during niraparib treatment for safety purposes. In the NOVA trial, the most common grade 3/4 AEs among niraparib-treated cases included thrombocytopenia (33.8\%), anemia (25.3\%), and neutropenia (19.6\%) (9); in the PRIMA trial, they also included anemia (31.0\%), thrombocytopenia (28.7\%), and neutropenia (12.8\%) (10). The phase 3 NORA trial showed grade $3 / 4$ neutropenia, thrombocytopenia, and anemia rates of $20.3 \%, 11.3 \%$, and $14.7 \%$, respectively, among Chinese patients with OC (11). Recently, a real-world study in the USA (12) that assessed 153 patients revealed that during the 3-month period following niraparib initiation, 57 (37\%) patients had at least one of the three most common all-grade AEs, which were fatigue (24\%; grade $3 / 4,3 \%)$, nausea $(16 \%$; grade $3 / 4,2 \%)$, and thrombocytopenia ( $14 \%$; grade $3 / 4,3 \%$ ). In older patients ( $\geq 70$ years of age) in the ENGOT-OV16/NOVA trial, the commonest grade 3/4 AEs included thrombocytopenia (34.4\%), neutropenia (16.4\%), and anemia (13.1\%) (16). These discrepancies in safety data highlight the need for real-world studies for more accurate representation of clinical reality $(13,14)$. In the present study, the rates of grade $\geq 1$ and $3 / 4$ anemia and thrombocytopenia were $34.9 \%$ and $7.0 \%$, and $39.5 \%$ and $11.6 \%$, respectively. These rates were generally lower than those reported in randomized controlled trials (9-11), and were closer to those observed in a real-life setting in the USA (12). Besides clinical trials, there is always a risk of under-reporting of grade $1 / 2 \mathrm{AEs}$, as they often do not require specific management or can be addressed by pharmacists or general clinics. Nevertheless, the rate of grade 3/4 AEs should be more accurate, as these AEs require specific management and often hospitalization, making them more likely to be reported to the treating oncologist. In clinical trials, patients are carefully selected, which can impact the incidence of AEs. Although hematological AEs must always remain a concern for oncologists, our results suggest that niraparib is safe and well-tolerated by many patients.

This study has limitations. First, it was a retrospective study with all inherent biases. Still, we included all patients treated with an ISD of niraparib during the study period. Second, all cases were treated in the same center, and the patients were small in number. Third, it was not possible to perform a multivariable analysis to identify patients at a high risk of hematological AEs. Fourth, since niraparib is a recently discovered molecule, no mid- or long-term followup data are available yet; however, future studies should examine AEs over the whole treatment course. Finally, because of the short follow-up, no analysis of efficacy was possible.

In conclusion, most hematological AEs in patients with advanced OC treated with an ISD of niraparib can be managed by dose modification and/or symptomatic treatment. Only $2.3 \%$ of patients in our study discontinued treatment. Niraparib with an ISD is a tolerable option for advanced OC. This real-world study has indicated that in Chinese clinical practice, the occurrence rate of grade $\geq 3$ hematological AEs (leukopenia, anemia, and thrombocytopenia) among patients with an ISD of niraparib (patients with a bodyweight $<77 \mathrm{~kg}$ or a platelet count $<150,000 / \mathrm{mL}$ received $200 \mathrm{mg} /$ day, and all other patients $300 \mathrm{mg} /$ day) is lower than that reported in the phase III NORA trial.

\section{Acknowledgments}

The authors thank all the physicians who provided medical 
records for this analysis.

Funding: None.

\section{Footnote}

Reporting Checklist: The authors have completed the STROBE reporting checklist. Available at http://dx.doi. org/10.21037/atm-21-2252

Data Sharing Statement: Available at http://dx.doi. org/10.21037/atm-21-2252

Conflicts of Interest: Both authors have completed the ICMJE uniform disclosure form (available at http://dx.doi. org/10.21037/atm-21-2252). The authors have no conflicts of interest to declare.

Ethical Statement: The authors are accountable for all aspects of the work in ensuring that questions related to the accuracy or integrity of any part of the work are appropriately investigated and resolved. All procedures performed in this study involving human participants were in accordance with the Declaration of Helsinki (as revised in 2013). The study was approved by institutional ethics board of Cancer Hospital of the University of Chinese Academy of Sciences (Zhejiang Cancer Hospital) (No. IRB-2021-71), and the requirement for informed consent was waived.

Open Access Statement: This is an Open Access article distributed in accordance with the Creative Commons Attribution-NonCommercial-NoDerivs 4.0 International License (CC BY-NC-ND 4.0), which permits the noncommercial replication and distribution of the article with the strict proviso that no changes or edits are made and the original work is properly cited (including links to both the formal publication through the relevant DOI and the license). See: https://creativecommons.org/licenses/by-nc-nd/4.0/.

\section{References}

1. Chen $\mathrm{W}$, Zheng R, Baade PD, et al. Cancer statistics in China, 2015. CA Cancer J Clin 2016;66:115-32.

2. NCCN Clinical Practice Guidelines in Oncology (NCCN Guidelines). Ovarian Cancer, Including Fallopian Tube Cancer and Primary Peritoneal Cancer. Version 2.2020. Fort Washington: National Comprehansive Cancer Network, 2021.

3. Colombo N, Sessa C, Bois AD, et al. ESMO-ESGO consensus conference recommendations on ovarian cancer: pathology and molecular biology, early and advanced stages, borderline tumours and recurrent disease. Int J Gynecol Cancer 2019. [Epub ahead of print]. doi: 10.1136/ ijgc-2019-000308.

4. Armstrong DK, Bundy B, Wenzel L, et al. Intraperitoneal cisplatin and paclitaxel in ovarian cancer. $\mathrm{N}$ Engl J Med 2006;354:34-43.

5. Arora N, Talhouk A, McAlpine JN, et al. Long-term mortality among women with epithelial ovarian cancer: a population-based study in British Columbia, Canada. BMC Cancer 2018;18:1039.

6. Rose M, Burgess JT, O'Byrne K, et al. PARP inhibitors: clinical relevance, mechanisms of action and tumor resistance. Front Cell Dev Biol 2020;8:564601.

7. Dziadkowiec KN, Gąsiorowska E, Nowak-Markwitz E, et al. PARP inhibitors: review of mechanisms of action and BRCA1/2 mutation targeting. Prz Menopauzalny 2016;15:215-9.

8. Scott CL, Swisher EM, Kaufmann SH. Poly (ADPribose) polymerase inhibitors: recent advances and future development. J Clin Oncol 2015;33:1397-406.

9. Mirza MR, Monk BJ, Herrstedt J, et al. Niraparib maintenance therapy in platinum-sensitive, recurrent ovarian cancer. N Engl J Med 2016;375:2154-64.

10. González-Martín A, Pothuri B, Vergote I, et al. Niraparib in patients with newly diagnosed advanced ovarian cancer. N Engl J Med 2019;381:2391-402.

11. Wu X, Zhu J, Yin R, et al. LBA29 Individualized starting dose of niraparib in Chinese patients with platinumsensitive recurrent ovarian cancer (PSROC): a randomized, double-blind, placebo-controlled, phase III trial (NORA). Ann Oncol 2020;31:S1160-1.

12. Gallagher JR, Heap KJ, Carroll S, et al. Real-world adverse events with niraparib $200 \mathrm{mg} /$ day maintenance therapy in ovarian cancer: a retrospective study. Future Oncol 2019;15:4197-206.

13. Kim HS, Lee S, Kim JH. Real-world evidence versus randomized controlled trial: clinical research based on electronic medical records. J Korean Med Sci 2018;33:e213.

14. Eichler HG, Pignatti F, Schwarzer-Daum B, et al. Randomized controlled trials versus real world evidence: neither magic nor myth. Clin Pharmacol Ther 2021;109:1212-8.

15. Berek JS, Matulonis UA, Peen U, et al. Safety and dose modification for patients receiving niraparib. Ann Oncol 2018;29:1784-92. Erratum in: Ann Oncol 2019;30:859. 
16. Fabbro M, Moore KN, Dørum A, et al. Efficacy and safety of niraparib as maintenance treatment in older patients ( $\geq 70$ years) with recurrent ovarian cancer: Results from the ENGOT-OV16/NOVA trial. Gynecol Oncol

Cite this article as: Wang J, Zhu J. Real-world hematological adverse events in Chinese patients with advanced ovarian cancer treated with an individualized starting dose of niraparib. Ann Transl Med 2021;9(10):869. doi: 10.21037/atm-21-2252
2019;152:560-7.

(English Language Editor: J. Reynolds) 